\title{
Rebuttal to letter to the editor
}

\section{Robert Boyles, Rebecca Fowler, Derek Ramsey, Erin Burrows}

\author{
Physical Therapy, University of Puget Sound, Tacoma, WA, USA
}

Dear Editor,

We have read the recent letter, "Trigger point dry needling: the data does not support broad applicability or robust effect," in critique of the research we conducted, which was published as, "Effectiveness of trigger point dry needling for multiple body regions: a systematic review." We appreciate the critical eye of the authors of this letter and the opportunity to engage in dialogue around clinically-relevant topics such as trigger point dry needling (TDN), as doing so is an important aspect of moving our field forward with integrity toward improved patient outcomes.

We are committed to presenting an unbiased report of the state of the highest-quality literature on TDN. The results of our study, as summarized in the conclusion statement in the body of our systematic review, reflect a clearly observable trend toward pain reduction as a result of TDN. The statement reads:

The majority of the highest-quality studies of TDN in the literature to date seem to indicate that TDN is effective for reducing pain and tenderness in multiple body regions, including the head, trunk, upper extremity, and lower extremity. Lack of consistency among the articles in this review in regards to patient recruitment, protocol, methodology, and outcome measures precludes the formation of any strong conclusions from the available data. ${ }^{2}$

We would like to clarify our understanding of the available data in light of some of the points made by the authors of the letter mentioned above. First, the authors stated that, "only $47 \%$ of included trials showed statistically significant decreases in pain when compared to sham or alternative treatments." This statement deemphasizes the efficacy of TDN by combining studies that use sham with those that use other interventions for comparison to arrive at the stated percentage. In fact, the majority of articles demonstrate statistically significant decreases in pain when compared to sham and baseline. Many of the "alternative treatments" to which they refer are other needling or manual interventions that are known to be effective in reducing pain. The importance in comparing two interventions in this way lies not only in effectiveness, but also in factors such as cost, safety, and side effects.

Correspondence to: Robert Boyles, Physical Therapy, University of Puget Sound, Tacoma, Wa, 98416, USA. Email: bboyles@pugetsound.edu
As stated in our review, while TDN does not appear to be superior to other needling interventions for the reduction of pain, these comparison studies provide valuable information about treatment options, which each contain different inherent risks. For example, studies by Ay et al. ${ }^{3}$ and Eroglu et al. ${ }^{4}$ compared TDN to trigger point lidocaine injection. There was no statistically significant difference between the injection and dry needling groups in either study, and both studies reported that TDN groups did achieve the minimal clinically important difference (MCID) for reduction in pain from baseline. These comparison studies truly highlight the need for a cost/benefit analysis of dry needling vs. pharmaceutical injection, and offer the suggestion that TDN may possibly provide the more viable option for some patients.

Second, the letter states that TDN is clinically irrelevant for measures of pain. As stated in our systematic review, the majority of articles met the MCID for pain reduction (reported on pain scale outcome measures), as compared to either baseline when no sham was present or to a sham group. Even more important than showing statistical significance is the applicability of the data in the clinical setting, which is why we looked at all of the articles that were used in our study to assess if those studies actually met the MCID for a reduction in pain.

Finally, the quality of literature surrounding TDN is also questioned in the letter mentioned above. In an effort to avoid misleading evidence presented by low-quality research, we included articles that scored at $6 / 10$ or greater on the PEDro scale. Even so, methodologic faults were found in all articles. In reporting our research, we felt it was our role to report the most important faults to the readers alongside specific and summarized results, so that readers may make informed clinical decisions based on the best available evidence. We therefore encourage readers to review our "Methodologic Considerations" section and take note of cautionary statements, such as those made in Clinical Relevance section:

While certain trends are identified in this review, caution should be taken when extrapolating these results to the clinical setting because comparisons are not exact, and limitations in methodological rigor exist. ${ }^{2}$ 
As both clinicians and researchers, we consider these limitations within the evidence-based practice model, which dictates that decision-making be based on evidence in the literature, clinical reasoning, and patient preference. ${ }^{5}$ We encourage readers to consider the evidence presented in this systematic review as one contributing component to a greater scope of evidence-based practice.

When read out of the context of the full article, we understand that the conclusion statement of our abstract may be misunderstood. We hope that the information above clarifies our findings and encourages readers to critically analyze the literature and utilize the evidence presented here within the context of clinical reasoning, patient preference and larger environmental context.

Thank you.

\section{References}

1 Venere K, Ridgeway K. Trigger point dry needling: the data does not support broad applicability or robust effect. J Man Manipulative Ther [Internet].

2 Boyles R, Fowler R, Ramsey D, Burrows E. Effectiveness of trigger point dry needling for multiple body regions: a systematic review. J Man Manipulative Ther [Internet]. 2015;2042618615Y.000. Available from: http://www.maneyonline.com/doi/10.1179/2042618615Y.0000000014

3 Ay S, Evcik D, Tur B. Comparison of injection method in myofascial pain syndrome a randomized controlled trial. J Clin Rheumatol. 2010;29:19-23.

4 Eroglu P, Yilmaz O, Bodur H, Ates C. A comparison of the efficacy of dry needling, lidocaine injection, and oral flurbiprofen treatments in patients with myofascial pain syndrome: a double-blind (for injection, groups only), Randomized Clinical Trial. Arch Rheum. 2013;28:38-46.

5 Jewell D. Guide to evidence-based physical therapy practice. Sudbury, MA: Jones \& Bartlet Learning LLC; 2011. 